\title{
Meta
}

Journal des traducteurs

Translators' Journal

\section{ARrizABAlAga, María Inés (2013: Semiótica de la cultura / Ecosemiótica / Biorretórica, (Traducido por Silvia N. BAREI, Ana Inés LEUNDA y Ariel GóMEz PONCE). Córdoba: Facultad de Lenguas, Universidad Nacional de Córdoba, 202 p.}

\section{Cristina Naupert}

Volume 62, numéro 1, avril 2017

URI : https://id.erudit.org/iderudit/1040479ar

DOI : https://doi.org/10.7202/1040479ar

Aller au sommaire du numéro

Éditeur(s)

Les Presses de l’Université de Montréal

ISSN

0026-0452 (imprimé)

1492-1421 (numérique)

Découvrir la revue

Citer ce compte rendu

Naupert, C. (2017). Compte rendu de [ARRIZABALAGA, María Inés (2013: Semiótica de la cultura / Ecosemiótica / Biorretórica, (Traducido por Silvia N. BAREI, Ana Inés LEUNDA y Ariel Gómez PONCE). Córdoba: Facultad de Lenguas, Universidad Nacional de Córdoba, 202 p.] Meta, 62(1), 231-233. https://doi.org/10.7202/1040479ar d'utilisation que vous pouvez consulter en ligne. 
traduction. Cercel nous montre comment l'introduction de méthodes de recherche empruntées aux sociologues des années 1970 est devenue un outil clé dans la recherche de cette transparence

Bref, l'étude de Cercel représente une somme de tous les aspects de l'évolution de l'herméneutique traductive, tant sur le plan diachronique: l'auteure nous révèle par exemple, des détails peu connus sur les méthodes de travail de Schleiermacher, que sur le plan synchronique: elle nous présente une étude exhaustive et parfaitement structurée des différents développements récents de cette approche, dont elle esquisse, avec une perspicacité à la fois surprenante et convaincante, les potentiels de développement à venir. Précisons qu'il s'agit d'une thèse de doctorat soutenue devant un jury de l'Université de Fribourg-en-Brisgau, garant de qualité et de sérieux dans la recherche, qui en fait un ouvrage incontournable pour qui souhaite évoquer l'approche herméneutique en traduction, intégrant la subjectivité et la créativité dans sa réflexion théorique.

La présentation étant d'ordre systématique dans la partie synchronique, il serait souhaitable que, dans une deuxième édition, une annexe permette par exemple de suivre l'évolution de la pensée de certains chercheurs ou encore les critiques émises à l'égard d'autres, faisant ainsi de ce livre un parfait ouvrage de référence.

Bernd Stefanink Universitatea Babeș-Bolyai, Cluj-Napoca, Roumanie

IOANA BǍLǍCESCU Universitatea din Craiova, Craiova, Roumanie

\section{RÉFÉRENCES}

Gerzymisch, Heidrun, dir. (2013): Translation als Sinngebung. Münster: LIT Verlag.

GuIlford, Joy Peter (1975): Creativity: A Quarter Century of Progress. In: Irving A. TAYLOR et Jacob W. Getzels, dirs. Perspectives in Creativity, Chicago: Aldine. 37-59.

Popper, Karl R. (1959): The Logic of Scientific Discovery. New York: Basic Books.

SIEver, Holger (2010): Übersetzen und Interpretation. Die Herausbildung der Übersetzungswissenschaft als eigenständige Disziplin im deutschen Sprachraum von 1960 bis 2000. Francfort: Peter Lang.
Arrizabalaga, María Inés (2013: Semiótica de la cultura / Ecosemiótica / Biorretórica, (Traducido por Silvia N. BAREI, Ana Inés Leunda y Ariel Gómez Ponce). Córdoba: Facultad de Lenguas, Universidad Nacional de Córdoba, 202 p.

La antología de textos seleccionada para esta obra, en una cuidada edición de la Dra. Arrizabalaga, contiene una serie de artículos del amplio espectro de la semiótica de la cultura que se adentran en los enfoques biosemiótico, ecosemiótico, biorretórico y ecorretórico. Sus autores: Jesper Hoffmeyer, Kalevi Kull, Stephen Pain, Frederik Stjernfelt, Marcel Danesi y Mijail Lotman publicaron entre 1998 y 2008 los artículos incluidos en esta antología, que busca conectar interdisciplinarmente los Estudios de Retórica con los de las diferentes ramas de la Semiótica, buscando el apoyo de las ciencias de la vida, a partir del siguiente planteamiento: "El orden de la cultura y las preguntas por lo humano. Retórica e imaginarios de la vida." En concreto, en dichos ensayos se reflexiona sobre la vinculación de la semiótica de la cultura con la biosemiótica o ecosemiótica, por un lado, y de la retórica de la cultura con la biorretórica, por otro.

El equipo de colaboradores, coordinado por la $\mathrm{D}^{\text {ra }}$ Arrizabalaga, formado por Silvia N. Barei, Ana Inés Leunda y Ariel Gómez Ponce, ha tenido el acierto de traducir los textos ensayísticos de los autores mencionados. Gracias a la mediación lingüística de los traductores, se ha puesto a disposición de los académicos de habla hispana este volumen contribuyendo así a la difusión de ideas en el campo de las ciencias humanísticas en su relación con las ciencias naturales, en general, y en el de la traductología en su más amplio espectro temático y conceptual. Igualmente se pone a disposición de los académicos de habla hispana unas investigaciones que, de no haber gozado del proceso de traslación, quizá no alcanzarían fuera del ámbito anglosajón la debida difusión académica y, consecuentemente, se pone a disposición de la comunidad científica auténticamente internacional -no guiada por una única lingua franca predominante- la reflexión sobre si debemos seguir guiándonos por conceptos aparentemente contrapuestos -mundo natural y mundo cultural-y un paradigma antropocéntrico o si, por el contrario, podemos también enfocar la mirada hacia un paradigma biocéntrico y tener una visión integradora de los diferentes ámbitos científicos. En definitiva, esta obra nos guía por las bondades de la interdisciplinariedad y la colaboración investigadora entre representantes de las ciencias naturales y de las ciencias humanísticas como unión natural y lógica para el avance del conocimiento. 
A continuación, pasaremos a destacar con unas breves pinceladas algunas de las ideas más sobresalientes y llamativas de cada uno de los artículos traducidos para esta antología. Gracias a la cuidada edición, realizada con esmero pensando en el mundo académico, el lector dispone de un aparato crítico en el que las notas a pie de página, algunas presentes en los textos de origen y otras aportadas por los respectivos traductores en los textos meta, facilitan la lectura y la comprensión de determinados conceptos de relativa cripticidad.

El primer artículo: «De animal a humano», firmado por Jesper Hoffmeyer, publicado originalmente por este autor en 2008 como uno de los capítulos de su obra Biosemiotics, comienza con una contundente afirmación sobre la presencia del talento para la semiosis no sólo en los seres humanos sino también en otros seres vivos, cuya comunicación es imprescindible en el proceso evolutivo. Si bien puede parecernos, a priori, una idea que, cuanto menos, causa extrañeza si trasladamos a un aspecto concreto del campo de la literatura y el arte lo que Hoffmeyer plasma en este capítulo, quizá nos parezca que su contundente afirmación es irrefutable. Pensemos, a título de ejemplo, en la obra Mitsou que el gran poeta de las letras alemanas Rainer Maria Rilke (1875-1926) ${ }^{1}$ escribió sobre la historia de un gato que, previamente, había plasmado gráficamente en cuarenta dibujos el pintor Balthus ${ }^{2}$. En este texto rilkeano se pone en paralelo el comportamiento del felino en relación con el ser humano, su mutua adaptación, y la comunicación entre ellos; ambos, a pesar de utilizar una "competencia semiótica» diferente, se comprenden. Así, en este texto, el animal responde a la afirmación aristotélica del «ser que tiene alma». A lo largo del artículo, Hoffmeyer, siguiendo las teorías del antropólogo americano Terrence Deacon, va desgranando sus ideas y su justificación científica, basada en la antropología y en la neurobiología evolutiva para concluir que tanto desde el punto de vista temático como teórico estas ideas se enmarcan en la biosemiosis. Ante un artículo tan complejo, resulta de gran utilidad la inclusión de un breve glosario que facilita al lector la comprensión de algunos de los conceptos expuestos por el autor. Sin embargo, se echa en falta la no presencia de una relación de referencias bibliográficas finales que podrían llevar al lector interesado a seguir indagando en aquellas citas que el autor hace a pie de página.

Por lo que respecta al artículo de Winfried Nöth, titulado «Ecosemiótica», comienza, al igual que las demás contribuciones -lo cual hay que agradecer a la buena labor de los editores-con un cuadro-guía sobre las nociones clave tratadas en el texto. De esta manera se nos aclara que el centro de interés de la ecosemiótica no reside en el «homo semioticus» sino en el "organismus semioticus» $\mathrm{y}$ que estudia las interrelaciones semióticas entre los organismos y su medio ambiente que dichos organismos ponen en funcionamiento por motivos de supervivencia. De nuevo resulta interesante una afirmación que nos conduce a relacionar las ideas de Nöth con las reflexiones del poeta expresionista alemán Rilke sobre la construcción del yo, las capas que construyen el mundo interior y su relación extrasensorial con el entorno. Según Nöth, «entre el organismo y su mundo interior existe un círculo funcional o complementariedad, según la cual el organismo no es resultado de su medio, sino que participa de él como su artífice y constructor». Nöth recurre, para fundamentar sus teorías, al biólogo alemán Jakob von Uexküll, quien acuñó el concepto de Umwelt o Medio Ambiente y que mantuvo correspondencia con el poeta alemán Rilke, vivamente interesado por la biología y la expresividad de los seres vivos e incluso de los objetos inanimados, lo que daría lugar al poema cumbre del Dinggedicht o poema-cosa, titulado Der Panther (La pantera) ${ }^{3}$. Consideramos que unos textos de tanta enjundia conceptual, si despiertan la capacidad heurística y la relación interdisciplinar con el ámbito de la literatura, por ejemplo, pueden resultar más atractivos como punto de partida para investigaciones en el campo de la traductología, en general, y de la tematología comparada o de la traducción literaria, en particular.

El tercer artículo, «Un apunte sobre biorretórica», lo firma Kalevi Kull, quien ya desde las primeras páginas aclara la diferencia entre la retórica de la biología y la biorretórica, ocupándose la primera de estudiar la retórica del discurso biológico, mientras que la segunda «trata de analizar el comportamiento expresivo de los organismos en términos de retórica primordial (inconsciente)» y admite en el «discurso» de los organismos la presencia de figuras retóricas que el autor califica de «biometáfora, biohipérbole y bioonomatopeya» (p. 97). Kalevi Kull es también el autor de otros dos artículos incluidos en esta antología: «Un signo no está vivo; el texto, sí» y «Hacia una biosemiótica con Iuri Lotman». En el primero, Kalevi, en sus disquisiciones terminológicas y su afán de precisar conceptualmente la relación entre semiosis y traducción como resultado de un proceso textual, lleva a conceder "vida propia» a dicho proceso, mientras que en el segundo centra la atención en los estudios que Lotman hiciera sobre la semiótica de la cultura y su relación con la biología. El artículo recoge de forma detallada las fuentes en las que se basó Lotman para desarrollar sus reflexiones.

Stephen Pain firma el artículo «De la biorretórica a la zoorretórica», cuyo contenido se conforma basándose en las reflexiones en torno a la comunicación entre humanos y animales 
construida sobre pilares argumentados para la resolución de conflictos, mientras que el segundo término del título alude a ese mismo proceso, pero en el que los interlocutores, siendo los mismos, traban una relación en la que los conceptos resultan útiles para el trabajo y la convivencia entre ellos. A Pain se le considera el padre de la biorretórica, disciplina nacida en la década de los noventa del siglo pasado.

El siguiente artículo, firmado por Frederik Stjernfelt, versa sobre las veintidós hipótesis básicas de la biosemiótica, disciplina fundada por Jesper Hoffmeyer, primer autor presente en la selección de textos de esta antología. El interés del texto de Sternfelt radica en la claridad de la exposición sobre las ideas fundamentales en las teorías que propugna Hoffmeyer, por lo que consideramos un acierto el haber incluido la traducción por parte del equipo editorial y traductor, cuya encomiable labor va más allá de la traducción de unos textos ensayísticos y científicos. El producto del trabajo de los traductores es ser un auténtico puente no sólo lingüístico-cultural, metáfora que siempre se menciona, sino también ser fuente documental para posteriores investigaciones.

Otro de los artículos clarificadores sobre las disciplinas tratadas en la antología es el que escribe Marcel Danesi, «Hacia una terminología estándar para la (bio)semiótica,». Este versa sobre la terminología básica para comprender el contenido de las ideas desarrolladas en la biosemiótica, justificándose en la frecuente inconsistencia terminológica que aqueja a la teoría semiótica y, consecuentemente, a las disciplinas derivadas de ella. El autor propone una serie de términos sustentados en la teoría de sistemas modelizantes que explica el comportamiento semiótico de las especies. De acuerdo con ello, propone los términos antroposemiótica, zoosemiótica y fitosemiótica.

El último de los textos seleccionado por la editora y el equipo traductor lo firma Mijail Lotman y lo titula «Umwelt y semiosfera». El autor comienza su exposición citando al ya mencionado Jakob von Uexküll y construye el contenido de su texto sobre la base de la semiótica cultural y las funciones de la semiosfera o "constructo compuesto por los textos, la razón y los sistemas modelizantes de una cultura».

Para finalizar, deseamos reiterar el valor del trabajo traductor, tanto del proceso como del producto, del equipo formado por la Dra. Silvia N. Barei, directora del grupo de investigación sobre Estudios de Retórica, Lic. Ana Inés Leunda y Prof. Ariel Gómez Ponce, coordinados por la editora de la antología Dra. María Inés Arrizabalaga. La labor mediadora de este equipo traductor amplía horizontes sobre disciplinas cuyas teorías, investigaciones y resultados podrían quedar oscurecidos en la vastísima comunidad científica mundial de habla española de no haber sido por su encomiable y difícil trabajo de traducción de textos ensayísticos.

Cristina NAUpert

Universidad Rey Juan Carlos, Madrid

\section{NOTAS}

1. Véase al respecto el estudio introductorio, en la edición crítica de Martino Alba, P. (2015), a la traducción de la obra narrativa cumbre de Rilke: Los apuntes de Malte Laurids Brigge, Madrid: Ed. Cátedra, col. Letras Universales.

2. Nombre artístico de Balthasar Klossowski.

3. Remitimos nuevamente al estudio introductorio citado en la primera nota a pie de página.

BAER, Brian James (2016): Translation and the Making of Modern Russian Literature. New York/ London: Bloomsbury, 213 p.

In the era of total globalization, people of different cultures and religions, living in different countries and speaking different languages will always seek for the means of preservation of their national identities. The feeling of belonging, being part of a particular community is, according to Maslow (Maslow 1943), one of the basic human needs. Driven by this need of socialization, a human being is prone to look for the sense of security among the people who speak the same language and who share the same values which are usually embedded in particular social regulations and cultural artifacts, sometimes reinforced by different systems of beliefs. In the contemporary world of globalization and multiculturalism, with the traditional ethnic states becoming more and more obsolete, with the boundaries between different nations and cultures becoming more and more transparent, and with national languages eventually surrendering to "Globish," people still struggle to preserve what makes them stand out as a nation and what constitutes the core of their national identities: their native language, their national historical and cultural heritage.

When it comes to preservation of national identities, cultures and languages, it seems obvious that the leading role here belongs to national literatures which can be seen as tribunes of "national spirit," metaphoric sanctuaries of what any particular community considers valuable. For the sake of preservation and promotion, the values get embedded into comprehensive literary forms and then passed from one generation to another. However, given that "communities are to be distinguished...by the style in which they are imagined" (Anderson 1991: 6), it becomes 